\title{
Dental Flat Panel Conebeam CT in the Evaluation of Patients with Inflammatory Sinonasal Disease: Diagnostic Efficacy and Radiation Dose Savings
}

\author{
C. Leiva-Salinas, L. Flors, P. Gras, F. Más-Estellés, P. Lemercier, J.T. Patrie, M. Wintermark, and L. Martí-Bonmatí
}

\begin{abstract}
BACKGROUND AND PURPOSE: CT is the imaging modality of choice to study the paranasal sinuses; unfortunately, it involves significant radiation dose. Our aim was to assess the diagnostic validity, image quality, and radiation-dose savings of dental conebeam CT in the evaluation of patients with suspected inflammatory disorders of the paranasal sinuses.
\end{abstract}

MATERIAL AND METHODS: We prospectively studied 40 patients with suspected inflammatory disorders of the sinuses with dental conebeam CT and standard CT. Two radiologists analyzed the images independently, blinded to clinical information. The image quality of both techniques and the diagnostic validity of dental conebeam CT compared with the reference standard CT were assessed by using 3 different scoring systems. Image noise, signal-to-noise ratio, and contrast-to-noise ratio were calculated for both techniques. The absorbed radiation dose to the lenses and thyroid and parotid glands was measured by using a phantom and dosimeter chips. The effective radiation dose for $\mathrm{CT}$ was calculated.

RESULTS: All dental conebeam CT scans were judged of diagnostic quality. Compared with CT, the conebeam CT image noise was $37.3 \%$ higher $(P<.001)$ and the SNR of the bone was $75 \%$ lower $(P<.001)$. The effective dose of our conebeam CT protocol was $23 \mu$ Sv. Compared with $\mathrm{CT}$, the absorbed radiation dose to the lenses and parotid and thyroid glands with conebeam $\mathrm{CT}$ was $4 \%, 7.8 \%$, and $7.3 \%$ of the dose delivered to the same organs by conventional CT $(P<.001)$.

CONCLUSIONS: Dental conebeam CT is a valid imaging procedure for the evaluation of patients with inflammatory sinonasal disorders.

ABBREVIATIONS: $C N R=$ contrast-to-noise ratio; $T L D=$ thermoluminescent dosimeter

C $\mathrm{T}$ is the criterion standard imaging technique for the evalua-

tion of adult patients with suspected sinonasal inflammatory disease. ${ }^{1}$ The use of CT has increased dramatically, and it is estimated that approximately 4 million CT scans of the sinuses are currently obtained each year in the United States. ${ }^{2}$

$\mathrm{CT}$ involves considerable ionizing radiation. It accounts for $10 \%$ of radiology procedures but represents approximately twothirds of the total medical radiation dose, ${ }^{3,4}$ and $2 \%$ of all cancers in the United States may be attributable to the radiation derived from CT studies. ${ }^{5}$ Patients in whom an inflammatory disorder of the paranasal sinuses is suspected are frequently young, ${ }^{1,6}$ and in

Received January 6, 2014; accepted after revision April 14.

From the Departments of Radiology and Medical Imaging (C.L.-S., L.F., F.M.-E., P.L., L.M.-B.) and Physics (P.G.), ERESA (F.M.-E.), and GIBI Research Group (L.M.-B.), Hospital Universitario y Politécnico la Fe, Valencia, Spain; and Departments of Radiology and Medical Imaging (C.L.-S., L.F., M.W.) and Public Health Sciences (J.T.P.), University of Virginia, Charlottesville, Virginia.

Please address correspondence to Carlos Leiva-Salinas, MD, Neuroradiology Division, Department of Radiology, Hospital Universitario y Politécnico la Fe, Bulevar Sur s/n, Torre F, Valencia, Spain; e-mail: carlosleivasalinas@gmail.com

http://dx.doi.org/10.3174/ajnr.A4019 this population, radiation-induced cancer risk is considerably higher. ${ }^{5}$ Besides carcinogenesis, CT of the paranasal sinuses increases the risk of radiation-induced cataracts because the lens of the eye is a highly radiosensitive organ enclosed in the scanning field. ${ }^{1,7,8}$

One approach to reduce such adverse effects is to decrease the CT-related radiation dose by adjusting downward the scanner settings that determine it. Prior studies ${ }^{1,4,6,8-11}$ have shown that, indeed, reducing the radiation dose by $75 \%$ does not significantly impact the diagnosis of sinonasal inflammatory diseases. A different approach would be to replace conventional CT with another technique. Dental conebeam CT is an emerging clinical technique. It uses a cone-shaped x-ray beam and an exceptionally radiosensitive flat panel detector to provide high-resolution images with a low radiation dose. ${ }^{12,13}$ At present, the clinical use of this technique is largely centered on the dental region, where it is considered more effective and economical than conventional CT. ${ }^{14}$ Conebeam CT systems have limited soft-tissue contrast discrimination, ${ }^{13}$ compared with conventional CT. This limitation represents the main barrier to the extension of conebeam of into 
diagnostic imaging. In the sinonasal region, this technique may not be appropriate for the evaluation of tumors or complicated sinusitis or critical evaluation of small changes in soft-tissue attenuation. The purpose of our investigation was to assess the diagnostic validity and image quality of dental conebeam CT compared with standard CT in the evaluation of inflammatory disorders of the paranasal sinuses and to investigate the potential radiation-dose savings achieved with this technique.

\section{MATERIALS AND METHODS Study Patients and Protocol}

We prospectively enrolled all consecutive adult patients (older than 18 years of age) who were referred by the ear, nose, and throat clinic to our department to perform noncontrast CT of the paranasal sinuses due to a clinical suspicion of an inflammatory disorder of the nasal fossae or paranasal sinuses during a 3-month period. Although we had planned to exclude patients in whom a sinonasal neoplasia was discovered, no patient was excluded because we did not find any. CT was performed first. In the hour following the CT, an additional dental conebeam CT scan was obtained.

The institutional ethics committee approved our research protocol. All participants gave written informed consent.

\section{Image Acquisition}

The CT studies were performed on a 64-channel multidetector CT system (Brilliance 64; Philips Healthcare, Best, the Netherlands). We used the following protocol: $120 \mathrm{kV}, 120 \mathrm{mAs}, 64 \times$ $0.625 \mathrm{~mm}$ collimation, 0.75 -second rotation time, table feed of 0.7 $\mathrm{mm}$ (pitch factor of 0.891 ), and FOV of $18 \mathrm{~cm}$. This protocol is provided by the manufacturer for this specific diagnostic purpose. No iterative reconstruction technique was used because it is not available in our scanners. All raw datasets were reconstructed at 1 -mm section thickness in $0.5-\mathrm{mm}$ increments by using a routine bone reconstruction algorithm. On the basis of the transverse images, coronal reformations were reconstructed at $1-\mathrm{mm}$ section thicknesses.

The conebeam CT studies were performed on a dental conebeam CT (i-CAT Next Generation; Imaging Sciences International, Hatfield, Pennsylvania). We used the following protocol: $120 \mathrm{kV}, 5 \mathrm{~mA}, 0.4$-mm collimation, 13-cm scan length, 4.8second rotation time, $190^{\circ}$ tube rotation, 159 basis images, and FOV of $16 \mathrm{~cm}$. Because the system is not designed to image the sinuses, there is no manufacturer-recommended protocol for that purpose; we considered this combination of parameters adequate on the basis of our experience with dental studies. Raw images were reconstructed at $1-\mathrm{mm}$ section thicknesses in $0.5-\mathrm{mm}$ increments. Coronal reformations were reconstructed at $1-\mathrm{mm}$ section thicknesses in 1-mm increments.

\section{Image Analysis}

Two experienced radiologists (C.L.-S. and F.M.-E.) with 4 and 15 years of experience in reading head and neck studies, respectively, assessed the images independently. They were blinded to the clinical information provided in the CT request. In a first session, the studies were randomized and the radiologists evaluated the image quality of conventional and conebeam CT by using 2 different scoring methods.

Scoring method 1 is a previously published system ${ }^{1}$ that takes into account 6 anatomic structures: the osteomeatal unit, uncinate process, infundibulum, frontal recess, attachments of the middle turbinate, and path of the optic nerve. For each structure, the following scores were assigned, depending on how well it was visualized: 0 , not demonstrated; 1 , demonstrated but not clearly visualized; 2 , clearly visualized. The right and left sides were analyzed separately. The maximum score for each of the left and right sides was 12. For analysis purposes, we calculated the mean of the scores of the right and left sides combined.

Scoring method 2 is based on another previously published grading system. ${ }^{8}$ It takes into account the radiologist's subjective impression of the overall quality of the study regarding the evaluation of the bony structures described in scoring method 1 by using a 5-point scale: 1 , unacceptable noise rendering a study nondiagnostic; 2 , low noise resulting in diagnostic uncertainty; 3 , average image quality with a correct diagnosis being highly likely; 4 , good image quality enabling a confident diagnosis; 5 , excellent image quality of the best diagnostic value.

In a separate session, the same radiologists evaluated the diagnostic validity of dental conebeam CT compared with conventional CT by using scoring method 3 , in which we randomized the studies and assigned the following scores: 0, not sufficient for diagnosis; 1 , sufficient for diagnosis.

\section{Image Noise and Signal-to-Noise and Contrast-To-Noise Ratios}

Image noise, signal-to-noise ratio, and contrast-to-noise ratio (CNR) were used to characterize image quality. These measurements were obtained by a single radiologist (P.L.). The attenuation in the cortical bone of the zygomatic arch, the pterygoid muscles, and air in the oropharynx were measured by drawing circular regions of interest for both CT and dental conebeam CT, according to the patient's individual anatomy. The diameter of the region of interest was made as large as possible, depending on the anatomic region. ROIs of similar size were placed at the same location on both imaging modalities. Image noise $(N)$ was calculated as the SD of the voxel values of the region of interest placed in the air. SNR of the bone was calculated by using the following equation:

$$
S N R_{\mathrm{b}}=A_{\mathrm{b}} / N
$$

where $A_{\mathrm{b}}$ is the mean attenuation of the voxel values of the region of interest placed in the bone and $N$ is the image noise. The contrast between the bone and the muscle-to-noise ratio $\left(C N R_{(\mathrm{b} / \mathrm{m})}\right)$ was calculated by using the following equation:

$$
C N R_{(\mathrm{b} / \mathrm{m})}=\left(A_{\mathrm{b}}-A_{\mathrm{m}}\right) / N
$$

where $A_{\mathrm{b}}$ and $A_{\mathrm{m}}$ are the mean attenuation of the voxel values of the region of interest placed in the bone and muscle respectively, and $N$ is the image noise. $C N R_{(\mathrm{b} / \mathrm{a})}$ (bone and air) and $C N R_{(\mathrm{m} / \mathrm{a})}$ (muscle and air) were calculated similarly.

AJNR Am J Neuroradiol 35:2052-57 Nov 2014 www.ajnr.org 


\section{Radiation-Dose Analysis}

The absorbed radiation dose to the lens of the eye and thyroid and parotid glands was measured by using an adult male skull and a tissue-equivalent phantom (radiation analog dosimetry system; Nuclear Associates, Hicksville, New York). Lithium fluoride thermoluminescent dosimeter (TLD) chips (EXT-RAD, XD-100; Harshaw, Cleveland, Ohio), $3.2 \times 3.2 \times 0.4 \mathrm{~mm}$, were used to record the absorbed radiation dose at selected locations in the head and neck region of the phantom. The chips were precalibrated for diagnostic $\mathrm{x}$-ray energies with an accuracy and precision of $5 \%$. At the start of each CT or dental conebeam CT scan, the TLD chips were superficially placed over the right and left lenses of the eye, the left parotid gland, and the thyroid surface. We measured the dose delivered to both lenses, because those were specific targets of interest. TLD doses were assumed equal to the dose delivered to each of the organs. Three scan acquisitions, each by using a different set of 4 TLD chips, were performed to provide a more reliable measure of radiation, and the mean was calculated for each TLD position.

The CT effective dose was calculated from the dose-length product (DLP) recorded from each CT protocol. A normalized conversion factor $(k)$ of 0.0023 for the head was used to calculate the effective dose by using the following formula ${ }^{15}$ :

$$
E D(\mathrm{mSv})=D L P(\mathrm{mGy} \times \mathrm{cm}) \times k[\mathrm{mSv} /(\mathrm{mGy} \times \mathrm{cm})] .
$$

Doses from TLDs at the different positions within tissue or organ were used to express the tissue-absorbed dose in milligrays. The products of these values and the percentage of a tissue or organ irradiated for the dental conebeam CT examination were used to calculate the equivalent dose $(E q D)$ in millisieverts. The proportion of the different tissues in the head and neck region, directly exposed to radiation (as a percentage of the total body), was set on the basis of evidence from previous studies. ${ }^{16,17}$ The effective dose was calculated by using the following formula ${ }^{16,17}$ :

$$
E D(\mathrm{mSv})=E q D(\mathrm{mSv}) \times W_{\mathrm{T}},
$$

where $E q D$ is the equivalent dose and $W_{\mathrm{T}}$ is a tissue-weighting factor that represents the relative contribution of that organ or tissue to the overall carcinogenic risk.

\section{Statistical Analysis}

The differences in image quality and diagnostic validity scores were compared by using Wilcoxon signed rank tests. The interreader agreement for such scores for both conventional CT and conebeam CT and the intrareader agreement for conventional CT versus conebeam CT were quantified by using a linear-weighted $\kappa$ analysis.

The differences in effective radiation doses between CT and dental conebeam CT were analyzed by using paired $t$ tests. The absorbed radiation data were analyzed by using a linear-mixed model. The sources of variation analyzed were the imaging technique (CT, dental conebeam CT) and the anatomic organ (left lens, right lens, right parotid gland, thyroid gland). The null hypothesis decision rule was based on a $P \leq .05$ criterion with a Bonferroni correction for 4 hypothesis tests.

The difference in image noise between CT and dental

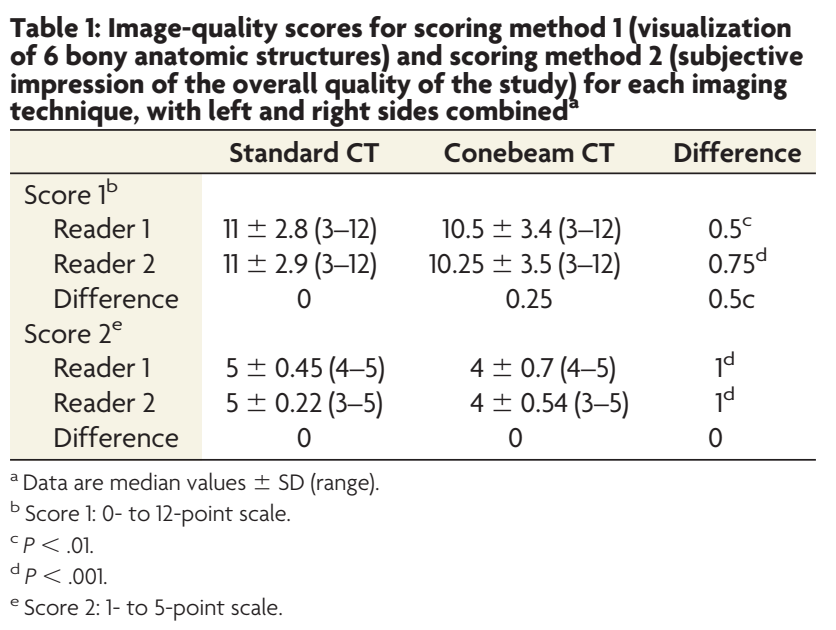

Table 2: Interreader agreement regarding image-quality scores for CT and conebeam CT

$\kappa$ Statistics $(95 \% \mathrm{Cl})$

CT

Score $1^{a}$

$0.78(0.60-0.80)$

Score $2^{b}$

$0.08(-0.16-0.32)$

Conebeam CT

Score $1^{\mathrm{a}}$

$0.79(0.70-0.87)$

Score $2^{b}$

$0.40(0.22-0.59)$

a Score 1: 0- to 12-point scale.

${ }^{\mathrm{b}}$ Score 2: 1- to -5-point scale.

Table 3: Intrareader agreement between CT and conebeam CT image-quality scores for each reader

\begin{tabular}{ll}
\hline & $\kappa$ Statistics $(95 \% \mathrm{Cl})$ \\
\hline Reader 1 & \\
Score $1^{\mathrm{a}}$ & $0.82(0.75-0.90)$ \\
Score 2 & \\
Reader 2 & $0.03(-0.05-0.12)$ \\
Score $1^{\mathrm{a}}$ & $0.70(0.60-0.80)$ \\
Score 2 $^{\mathrm{b}}$ & $0.01(-0.00-0.01)$ \\
\hline
\end{tabular}

a Score 1: 0- to 12-point scale.

b Score 2: 1- to 5-point scale.

conebeam CT was compared by using Wilcoxon signed rank testing.

The statistical software packages SAS, Version 9.2.2 (SAS Institute, Cary, North Carolina) and Spotfire S+, Version 8.1 (TIBCO, Palo Alto, California) were used.

\section{RESULTS}

We enrolled 40 consecutive patients (mean age, $44 \pm 15$ years; range, $18-82$; female, $57.5 \%)$. Sixteen patients (40\%) were younger than 40 years of age.

The median score for method 1, regarding identification of anatomic structures, was 11 for standard CT and 10.25 for dental conebeam CT $(P<.01)$ (Table 1$)$. Regarding scoring method 1 , the average specific scores for the osteomeatal unit, uncinate process, infundibulum, frontal recess, attachments of the middle turbinate, and path of the optic nerve were 1.58, 1.52, 1.46, 1.58, 1.97, and 2 for CT, and 1.56, 1.37, 1.34, 1.44, 1.71, and 1.81 for conebeam CT. The median score for method 2, regarding the subjective quality of the study, was 5 for CT and 4 for dental conebeam CT $(P<.01)$ (Table 1). Regarding scoring method 3, both readers judged all the dental conebeam CT studies valid for 
Table 4: Image noise, signal-to-noise ratio, and contrast-to-noise ratio for each imaging technique ${ }^{\mathrm{a}}$

\begin{tabular}{lccccc}
\hline \multicolumn{1}{c}{ Noise } & SNR $_{\mathrm{b}}$ & $\mathbf{C N R}_{(\mathrm{b} / \mathrm{m})}$ & $\mathbf{C N R}_{(\mathrm{b} / \mathrm{a})}$ & $\mathbf{C N R}_{(\mathbf{m} / \mathrm{a})}$ \\
\hline CT & $29.28 \pm 9.39$ & $100.35 \pm 33.1$ & $97.03 \pm 31.63$ & $163.80 \pm 54.81$ & $66.76 \pm 23.31$ \\
Conebeam CT & $46.76 \pm 15.84$ & $25.33 \pm 16.73$ & $28.45 \pm 18.11$ & $50.46 \pm 31.9$ & $22 \pm 14$ \\
Difference & $17.48^{\mathrm{b}}$ & $75.02^{\mathrm{b}}$ & $68.58^{\mathrm{b}}$ & $113.34^{\mathrm{b}}$ & $44.76^{\mathrm{b}}$ \\
\hline
\end{tabular}

Note: $-\mathrm{SNR}_{\mathrm{b}}$ indicates SNR of the bone; $\mathrm{CNR}_{(\mathrm{b} / \mathrm{m})}$, contrast between the bone and the muscle-to-noise ratio; $\mathrm{CNR}_{(\mathrm{b} / \mathrm{a})}$, contrast between bone and air; $\mathrm{CNR}_{(\mathrm{m} / \mathrm{a})}$, $\mathrm{Contrast}$ between muscle and air.

${ }^{\text {a }}$ Data are median values $\pm \mathrm{SD}$.

$\mathrm{b} P<.001$.

Table 5: Absorbed radiation dose to the lenses of the eye and parotid and thyroid glands for each imaging technique ${ }^{a}$

\begin{tabular}{lcccc}
\hline & $\begin{array}{c}\text { Absorbed Dose Right } \\
\text { Lens (mGy) }\end{array}$ & $\begin{array}{c}\text { Absorbed Dose Left } \\
\text { Lens (mGy) }\end{array}$ & $\begin{array}{c}\text { Absorbed Dose Right Parotid } \\
\text { Gland (mGy) }\end{array}$ & $\begin{array}{c}\text { Absorbed Dose Thyroid } \\
\text { Gland (mGy) }\end{array}$ \\
\hline CT & $9.25 \pm 1.69$ & $9.69 \pm 1.08$ & $8.87 \pm 1.68$ & $0.45 \pm 0.13$ \\
Conebeam CT & $0.28 \pm 0.01^{b}$ & $0.4 \pm 0.03^{b}$ & $0.69 \pm 0.05$ & $0.05 \pm 0.05$ \\
Difference & $8.97^{\mathrm{c}}$ & $9.29^{\mathrm{c}}$ & $8.18^{\mathrm{c}}$ & $0.4^{\mathrm{c}}$ \\
\hline
\end{tabular}

${ }^{\mathrm{a}}$ Data are mean values \pm SD.

${ }^{b}$ For Conebeam CT, the absorbed radiation dose to the left lens was significantly higher than the dose to the right lens because the $\mathrm{x}$-ray tube describes a $190^{\circ}$ rotation around the left side of the patient's face.

${ }^{c} p<.001$
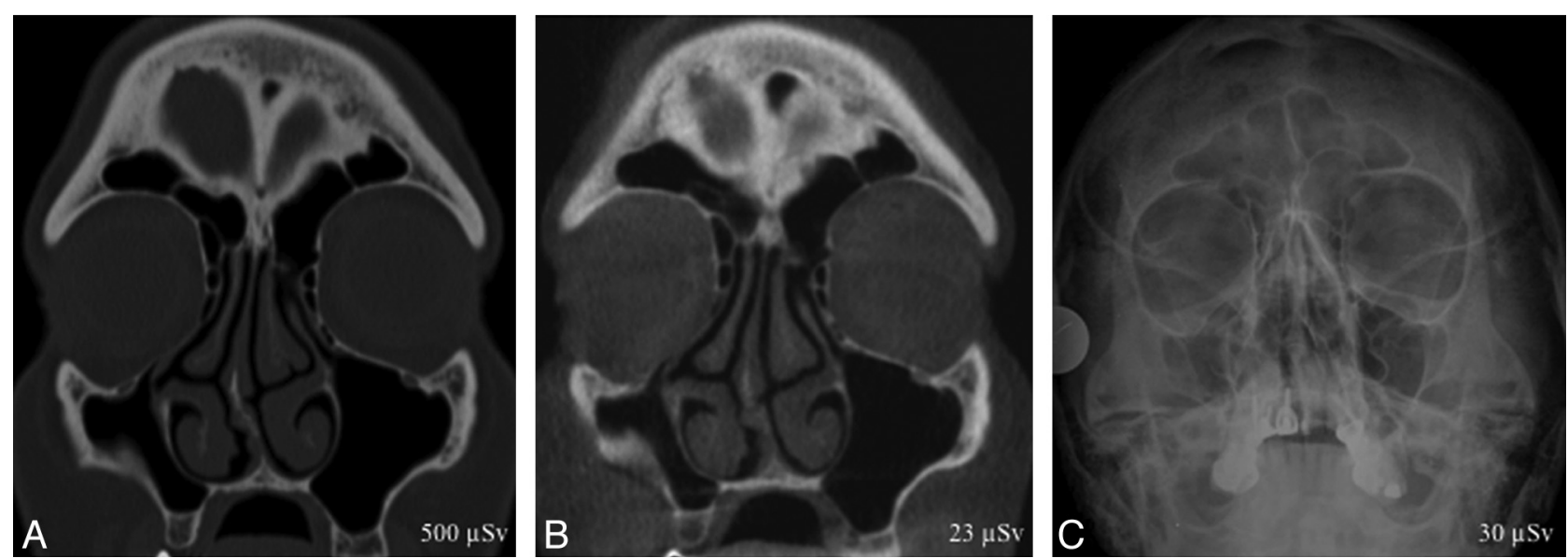

FIG 1. A 50-year-old woman with vague facial pain for several months and enophthalmos on physical examination. CT $(A)$ and conebeam $C T(B)$ coronal images show inward retraction of the right maxillary walls and apposition of the right uncinate process into the orbital floor, occluding the maxillary sinus infundibulum. The clinical and CT imaging findings were characteristic of silent sinus syndrome. CT scans delineate anatomic structures and demonstrate disease not shown on the $\mathrm{x}$-ray $(C)$. X-ray shows a small right maxillary sinus but is unable to help in the diagnostic work-up of the patient.

diagnosis. Tables 2 and 3 show the inter- and intrareader agreement for both imaging techniques.

Compared with conventional CT, the dental conebeam CT image noise was $37.3 \%$ higher $(P<.001)$, the SNR of the bone was $75 \%$ lower $(P<.001)$, and the CT $\mathrm{CNR}_{(\mathrm{b} / \mathrm{m})}, \mathrm{CNR}_{(\mathrm{b} / \mathrm{a})}$, and $\mathrm{CNR}_{(\mathrm{m} / \mathrm{a})}$ were $70 \%, 69.1 \%$, and $67 \%$ lower, respectively $(P<$ .001) (Table 4).

The mean effective radiation dose for CT was $0.511 \pm 0.039$ $\mathrm{mSv}$ (range, $0.44-0.61 \mathrm{mSv}$ ). The calculated effective radiation dose for dental conebeam CT was $0.023 \mathrm{mSv}$. Compared with CT, the dental conebeam CT effective radiation dose was $95.5 \%$ lower $(P<.001)$. Compared with CT, the dental conebeam CT absorbed radiation doses to the right lens, left lens, right parotid gland, and thyroid gland were 95.8\%, 97\%, 92.2\%, and $92.7 \%$ lower, respectively $(P<.001)$ (Table 5$)$.

A representative image from each technique is shown in Fig 1.

\section{DISCUSSION}

Our study suggests that by using conebeam CT technology, it is possible to drastically reduce the radiation dose without compro- mising the diagnostic capability in screening examination of the paranasal sinuses. While conventional CT better delineated the bony anatomic structures and obtained better objective metrics of image quality (lower image noise, higher SNR and CNR), the overall diagnostic validity of both imaging techniques was considered similar. Conebeam CT image quality was graded as good, compared with excellent for CT, enabling a confident diagnosis. Although CT evaluated the bony structures statistically better than conebeam CT, most bony anatomic structures were perfectly visualized with conebeam CT. That the image quality for CT was graded as excellent and that the SNR and CNR were significantly better for CT than for conebeam CT are evidence that the radiation dose used in the multidetector CT protocol was excessively high, as mentioned in the "Discussion."

The effective dose of our dental conebeam CT protocol was $23 \mu \mathrm{Sv}$, lower than the radiation dose from a sinus $\mathrm{x}$-ray, which is typically around $30 \mu \mathrm{Sv} .{ }^{18}$ As a reference, the natural background radiation dose is estimated to be $2280 \mu \mathrm{Sv}$ per year. ${ }^{19}$

Conebeam CT is an emerging technology with its main clinical 
application to date being dental imaging. Efficient use of the $\mathrm{x}$-ray beam in conebeam CT produces a relatively low $\mathrm{x}$-ray tube power requirement - as modest as $5 \mathrm{mAs}$ in our system — and therefore a low radiation dose. ${ }^{12,13}$ A prior study proposed a conebeam CT protocol to image the paranasal sinuses and studied the theoretic, potential radiation-dose savings of such a protocol. ${ }^{20}$ Other authors have investigated how often clinically important findings would be missed if conebeam CT were used routinely for sinus imaging by creating a "theoretic conebeam CT" from the original CT images. ${ }^{21}$ However, to our knowledge, no previous research has analyzed the diagnostic validity and potential radiation dose savings of dental conebeam CT compared with conventional CT in the evaluation of the paranasal sinuses, in a real clinical scenario.

Imaging of the sinuses involves imaging 3 tissues with considerably different attenuation: air, bone, and soft tissues. The inherent and naturally high contrast among these structures, coupled with CT technologic developments, has favored the use of lowdose CT protocols without losing diagnostic power, pushing the limits of the radiation-dose reduction by a factor of $>25$ in the past 25 years. $^{4,10,11,22}$

A large number of prior studies have successfully achieved reduction of the CT-related radiation dose by either adjusting the acquisition settings that determine the radiation dose, ${ }^{1,4,6,8-11}$ the image reconstruction algorithms, ${ }^{23}$ or by using the latest available CT systems. ${ }^{24}$ The lowest published effective radiation dose for a sinus CT protocol in the clinical setting was $47 \mu \mathrm{Sv}$, very low, but more than double that with conebeam $\mathrm{CT} .{ }^{11}$ A recent study using state-of-the-art CT technology with the lowest possible radiation dose in postmortem heads yielded an absorbed dose of $0.64 \mathrm{mGy}$ for the lens of the eye and $0.085 \mathrm{mGy}$ for the thyroid gland, ${ }^{19}$ still $88 \%$ and $70 \%$ more radiation doses, respectively, than the results obtained with our dental conebeam CT protocol.

Patients with inflammatory disorders of the paranasal sinuses are often pediatric or young adults, ${ }^{1,6}$ and in this population, radiation-dose reduction is particularly important, ${ }^{5}$ especially considering that for chronic sinonasal disorders, serial scanning is often performed for follow-up; hence, patients are subject to a cumulative radiation exposure. $^{8}$

From an economic perspective, a CT scan of the sinuses has an average cost of $\$ 875$; for a dental conebeam $\mathrm{CT}$, it is around $\$ 160-\$ 275 .^{25,26}$

This study had certain limitations. First, it may have been biased toward dental conebeam CT because the readers were able to identify whether the images they were scoring were acquired either with conebeam CT or with a standard CT system, due to the higher noise and lower soft-tissue definition of the images acquired with conebeam CT. Second, the study was limited to adult patients with sinonasal inflammatory conditions. The generalization of our results to all patients who are referred to a radiology department for a CT scan of the sinuses may be problematic. Additionally, a number of pathologic entities may indeed require a soft-tissue algorithm for diagnostic evaluation (sinonasal and oral cavities and oropharyngeal neoplasms). Dental conebeam CT is not reliable for assessing the soft tissues ${ }^{13}$ and should probably only be used to screen for uncomplicated inflammatory mu- cosal disease. Investigation of patients with noninflammatory diseases was beyond the scope of our article.

Third, the imaging protocol installed as standard on our CT system and the one we used in our investigation are not low-dose protocols. They use a tube current of $120 \mathrm{mAs}$ rather than the sufficient $50 \mathrm{mAs}$ recommended by the literature. ${ }^{1}$ If we had used a low-dose protocol, the radiation-dose savings of dental conebeam CT would have been proportionately lower in comparison with standard CT. Despite this possible outcome, the effective and absorbed radiation doses obtained with dental conebeam CT compared very favorably with those in all other previous studies and cannot be achieved with the currently available CT scanners. Using a standard-dose CT protocol also ensures that we compared dental conebeam CT with an optimal criterion standard, because there are no published data about the diagnostic quality of a low-dose protocol acquired with our CT system.

\section{CONCLUSIONS}

Due to its image quality, low radiation dose, and low economic cost, dental conebeam CT is a valid first-line imaging test for the evaluation of patients with suspected inflammatory sinonasal disorders. It allows a reduction in radiation dose by a factor of 25 compared with standard CT without a low-dose protocol implemented. Conebeam CT provides mostly bone information; due to its limited soft-tissue contrast discrimination, it may not be appropriate for imaging patients with suspected complicated sinusitis.

\section{ACKNOWLEDGMENTS}

We thank Carmen Argudo, RN, and all the CT technologists for their enthusiasm and wonderful work and Emilio Casal, $\mathrm{PhD}$, from the Centro Nacional de Dosimetría, for providing the TLD chips and assisting in the radiation-dose calculation.

Disclosures: Max Wintermark—RELATED: Grants/Grants Pending: GE Healthcare and Philips Healthcare. * *Money paid to the institution.

\section{REFERENCES}

1. Sohaib SA, Peppercorn PD, Horrocks JA, et al. The effect of decreasing $\mathrm{mAs}$ on image quality and patient dose in sinus CT. Br J Radiol 2001;74:157-61

2. Conference of Radiation Control Program Directors, Department of Health and Human Services (2006). What's NEXT? Nationwide Evaluation of X-ray Trends: 2000 computed tomography. http://www. crcpd.org/Pubs/NEXT_docs/NEXT2000-CT.pdf. Accessed May 24, 2014

3. Mettler FA Jr, Wiest PW, Locken JA, et al. CT scanning: patterns of use and dose. J Radiol Prot 2000;20:353-59

4. Mulkens TH, Broers C, Fieuws S, et al. Comparison of effective doses for low-dose MDCT and radiographic examination of sinuses in children. AJR Am J Roentgenol 2005;184:1611-18

5. Brenner DJ, Hall EJ. Computed tomography: an increasing source of radiation exposure. $N$ Engl J Med 2007;357:2277-84

6. Nauer CB, Eichenberger A, Dubach P, et al. CT radiation dose for computer-assisted endoscopic sinus surgery: dose survey and determination of dose-reduction limits. AJNR Am J Neuroradiol 2009;30:617-22

7. Dammann F, Momino-Traserra E, Remy C, et al. Radiation exposure during spiral-CT of the paranasal sinuses [in German]. Rofo 2000;172:232-37

8. Brem MH, Zamani AA, Riva R, et al. Multidetector CT of the para- 
nasal sinus: potential for radiation dose reduction. Radiology 2007;243:847-52

9. Kropil $\mathrm{P}$, Cohnen M, Andersen K, et al. Image quality in multidetector CT of paranasal sinuses: potential of dose reduction using an adaptive post-processing filter [in German]. Rofo 2010;182:973-78

10. Marmolya G, Wiesen EJ, Yagan R, et al. Paranasal sinuses: low-dose CT. Radiology 1991;181:689-91

11. Tack D, Widelec J, De Maertelaer V, et al. Comparison between lowdose and standard-dose multidetector CT in patients with suspected chronic sinusitis. AJR Am J Roentgenol 2003;181:939-44

12. Gupta R, Cheung AC, Bartling SH, et al. Flat-panel volume CT: fundamental principles, technology, and applications. Radiographics 2008;28:2009-22

13. Miracle AC, Mukherji SK. Conebeam CT of the head and neck. Part 1. Physical principles. AJNR Am J Neuroradiol 2009;30:1088-95

14. Hashimoto K, Kawashima S, Araki M, et al. Comparison of image performance between cone-beam computed tomography for dental use and four-row multidetector helical CT. J Oral Sci 2006;48:27-34

15. Bongartz G, Golding S, Jurik A, et al. European guidelines on quality criteria for computed tomography. 2000; Publication no. EUR 16262 EN. http://www.drs.dk/guidelines/ct/quality/htmlindex.htm. Accessed May 24, 2014

16. Ludlow JB, Davies-Ludlow LE, Brooks SL. Dosimetry of two extraoral direct digital imaging devices: NewTom conebeam CT and Orthophos Plus DS panoramic unit. Dentomaxillofac Radiol 2003; 32:229-34

17. Ludlow JB, Ivanovic M. Comparative dosimetry of dental CBCT devices and 64-slice CT for oral and maxillofacial radiology. Oral Surg Oral Med Oral Pathol Oral Radiol Endod 2008;106:106-14

18. Wall BF, Hart D. Revised radiation doses for typical X-ray examinations: report on a recent review of doses to patients from medical X-ray examinations in the UK by NRPB - National Radiological Protection Board. Br J Radiol 1997;70:437-39

19. National Council on Radiation Protection and Measurements. NCRP Report No. 160, Ionizing radiation exposure of the population of the United States. National Council on Radiation Protection and Measurements. http://www.ncrponline.org/Publications/Press_Releases/ 160press.html. Accessed August 15, 2013

20. Güldner C, Ningo A, Voigt J, et al. Potential of dose reduction in cone-beam-computed tomography (CBCT) for radiological diagnostics of the paranasal sinuses. Eur Arch Otorhinolarygol 2013; 270:1307-15

21. Fakhran S, Alhilali L, Sreedher G, et al. Comparison of simulated cone beam computed tomography to conventional helical computed tomography for imaging of rhinosinusitis. Laryngoscope 2014;124:2002-06

22. Zinreich SJ, Kennedy DW, Rosenbaum AE, et al. Paranasal sinuses: CT imaging requirements for endoscopic surgery. Radiology 1987; 163:769-75

23. Bulla S, Blanke P, Hassepass F, et al. Reducing the radiation dose for low-dose CT of the paranasal sinuses using iterative reconstruction: feasibility and image quality. Eur J Radiol 2012;81:2246-50

24. Schulz B, Potente S, Zangos S, et al. Ultra-low dose dual-source highpitch computed tomography of the paranasal sinus: diagnostic sensitivity and radiation dose. Acta Radiol 2012;53:435-40

25. CT Maxillofacial (Sinus) Procedure and Cost Information. New Choice Health. 2013. http://www.newchoicehealth.com/Directory/Procedure/ 4/CT\%20Maxillofacial\%20(Sinus). Accessed October 25, 2013

26. Christell H, Birch S, Horner K, et al. A framework for costing diagnostic methods in oral health care: an application comparing a new imaging technology with the conventional approach for maxillary canines with eruption disturbances. Community Dent Oral Epidemiol 2012;40:351-61 